ARTICLE

DOI: $10.1038 /$ ncomms11983 OPEN

\title{
Strain-engineered diffusive atomic switching in two-dimensional crystals
}

Janne Kalikka ${ }^{1,2, \star \dagger}$, Xilin Zhou ${ }^{1, \star}$, Eric Dilcher ${ }^{3}$, Simon Wall ${ }^{3}$, Ju Li $^{2} \&$ Robert E. Simpson ${ }^{1}$

Strain engineering is an emerging route for tuning the bandgap, carrier mobility, chemical reactivity and diffusivity of materials. Here we show how strain can be used to control atomic diffusion in van der Waals heterostructures of two-dimensional (2D) crystals. We use strain to increase the diffusivity of $\mathrm{Ge}$ and $\mathrm{Te}$ atoms that are confined to $5 \AA$ thick $2 \mathrm{D}$ planes within an $\mathrm{Sb}_{2} \mathrm{Te}_{3}-\mathrm{GeTe}$ van der Waals superlattice. The number of quintuple $\mathrm{Sb}_{2} \mathrm{Te}_{3} 2 \mathrm{D}$ crystal layers dictates the strain in the GeTe layers and consequently its diffusive atomic disordering. By identifying four critical rules for the superlattice configuration we lay the foundation for a generalizable approach to the design of switchable van der Waals heterostructures. As $\mathrm{Sb}_{2} \mathrm{Te}_{3}-\mathrm{GeTe}$ is a topological insulator, we envision these rules enabling methods to control spin and topological properties of materials in reversible and energy efficient ways.

\footnotetext{
${ }^{1}$ Singapore University of Technology and Design (SUTD), 8 Somapah Road, Singapore 487372, Singapore. ${ }^{2}$ Massachusetts Institute of Technology (MIT), Cambridge, Massachusetts 02139, USA. ${ }^{3}$ Institut de Ciencies Fotoniques (ICFO), Barcelona Institute of Science and Technology, Castelldefels, Barcelona 08860, Spain. * These authors contributed equally to this work. † Present address: Department of Physics, Tampere University of Technology, Tampere, Finland (J.K.). Correspondence and requests for materials should be addressed to R.E.S. (email: robert_simpson@sutd.edu.sg).
} 
an der Waals (vdW) heterostructures composed from layers of two-dimensional (2D) atomic crystals ${ }^{1}$ provide unprecedented freedom to systematically design the properties of materials. Their properties are dependent on the sequence of the layers ${ }^{2}$, interlayer separation ${ }^{3}$ and the stress in the layers ${ }^{4}$. The crystal structure of $\mathrm{Sb}_{2} \mathrm{Te}_{3}$, which comprises of covalently bonded $\mathrm{Sb}-\mathrm{Te}$ quintuple-layer blocks and vdW interblock bonding, provides the framework to build $\mathrm{Sb}_{2} \mathrm{Te}_{3}-\mathrm{GeTe}$ vdW heterostructures. A systematic design methodology would accelerate the optimization of $\mathrm{Sb}_{2} \mathrm{Te}_{3}-\mathrm{GeTe}$ heterostructures, phase change materials and switchable topological insulators ${ }^{5}$. In this work we show that biaxial strain selectively destabilizes GeTe layers within the $\mathrm{Sb}_{2} \mathrm{Te}_{3}-\mathrm{GeTe}$ heterostructure and allows diffusive atomic disordering within the GeTe layers. In practice we demonstrate that this $\mathrm{Ge}-\mathrm{Te}$ diffusive switching is controlled by the thickness of the $\mathrm{Sb}_{2} \mathrm{Te}_{3}$ layers and confined to an interface just $5 \AA$ thick.

Strain engineering is possibly the foremost generalizable methodology used to design materials with bespoke properties. The most familiar example is in metal oxide semiconductor fieldeffect transistors (MOSFETs) ${ }^{6}$ where the Si channel is strained by a lattice mismatch between it and the surrounding material ${ }^{7}$. The stress in the Si crystal alters the mobility of charge carriers and increases the switching speed of the MOSFET. The discovery of super strong $2 \mathrm{D}$ materials has unleashed strain engineering from the sub-percent levels of strain that can be sustained in bulk crystals to the extraordinarily large strains of 11 and 20\%, which can be sustained in graphene ${ }^{8}$ and $\mathrm{MoS}_{2}$ (ref. 9), respectively. This has provided strain engineers with a new degree of freedom to create strain-tuneable electronic and optoelectronic materials $^{10}$.

Strain engineering provides a conceptually simple means to control the properties of materials at an atomic level. Furthermore, it is well suited to computer-aided atomistic design of materials using tools such as density functional molecular dynamics (DF/MD). In contrast, modelling doped materials require a large simulation cell and hundreds of atoms to reach the low dopant concentrations that are typically used in experiments. Consequently, simulating doped materials is extremely computationally expensive. Models of strained crystals do not suffer from this problem. It is, therefore, surprising that strain engineering has not been applied to the development of $\mathrm{Sb}_{2} \mathrm{Te}_{3}-\mathrm{GeTe}$ phase change materials. This may be due to the misbelief that reversible switching in these materials is only possible by a melt-quench process, which would cause strain relaxation. Recently, a number of demonstrations have shown that switching in $\mathrm{Sb}_{2} \mathrm{Te}_{3}-\mathrm{GeTe}$ materials is possible without melting the crystal structure. For instance, the optical properties of the $\mathrm{Ge}_{2} \mathrm{Sb}_{2} \mathrm{Te}_{5}$ alloy can be switched without transiting through the molten phase ${ }^{11}$, and without loss of long-range order ${ }^{12}$, suggesting that only a subset of atoms need to move to change the material properties. Moreover, non-melt crystal-crystal transitions are possible in $\mathrm{Sb}_{2} \mathrm{Te}_{3}-\mathrm{GeTe}$ vdW heterostructures ${ }^{13}$ and now, in this work, we show theoretically and experimentally that biaxial strain can be used to reduce the switching energy of $\mathrm{Sb}_{2} \mathrm{Te}_{3}-\mathrm{GeTe}$ vdW heterostructures.

Crystalline $\mathrm{Sb}_{2} \mathrm{Te}_{3}-\mathrm{GeTe}$ superlattice structures have been designed to promote the movement of interfacial $\mathrm{Ge}$ atoms through a plane of Te atoms ${ }^{13}$. In effect the interfacial Ge and Te layers exchange position-an atomic switch. Ideally this atomic transition reduces entropic losses leading to a high efficiency, low-energy switching process ${ }^{14}$. After switching $\mathrm{Sb}_{2} \mathrm{Te}_{3}-\mathrm{GeTe}$ superlattices, the electrical resistivity increases by several orders of magnitude yet the superlattice remains crystalline. This led to the suggestion that the material undergoes a crystal-crystal transition $^{13}$. It is important to realize that this switching concept

is fundamentally different to that in $\mathrm{GeTe} / \mathrm{Sb}_{2} \mathrm{Te}_{3}$ superlattice-like structures (SLL) ${ }^{15}$. Both the GeTe and $\mathrm{Sb}_{2} \mathrm{Te}_{3}$ layers within the as-deposited SLL are relatively thick amorphous films that crystallize at different temperatures ${ }^{16}$ through three-dimensional diffusion of atoms into a system of polycrystalline ${ }^{17}$ layers without preferred orientation. The SLL's switching energy reduction stems from its low thermal conductivity ${ }^{15}$ and associated heating efficiency, not from controlled interfacial atomic diffusion.

The aim of this work is to establish a set of general rules that can be used to design and optimize phase transitions in vdW superlattices. Others have tried to further optimize switching in $\mathrm{Sb}_{2} \mathrm{Te}_{3}$-based superlattices by changing the composition of the other superlattice layers ${ }^{18-20}$, but the effects of doping and compositional tuning are difficult to predict and establishing general conclusions is not possible. In contrast, we model and experimentally demonstrate strain engineering of the $\mathrm{Sb}_{2} \mathrm{Te}_{3}-\mathrm{GeTe}$ superlattice such that the GeTe layers disorder at a temperature substantially lower than the melting temperature of the whole superlattice. Strain engineering is a more general approach to materials design than compositional tuning. Moreover, the approach can in principle be used to add switching function to vdW superlattices composed of other $2 \mathrm{D}$ crystals.

\section{Results}

Activation energy for switching. Recently Yu and Robertson ${ }^{21}$ showed that the activation energy for switching of Ge atoms in

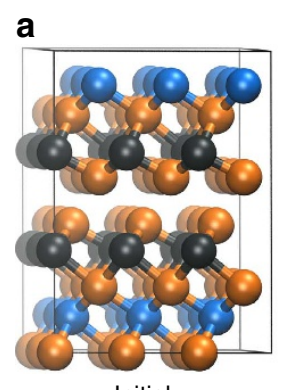

Initial

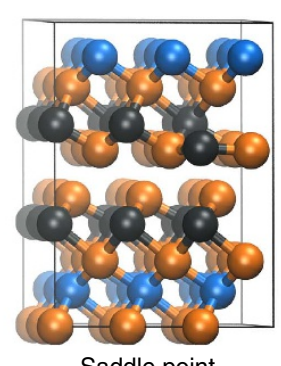

Saddle point

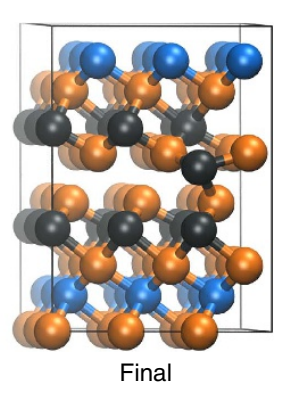

b

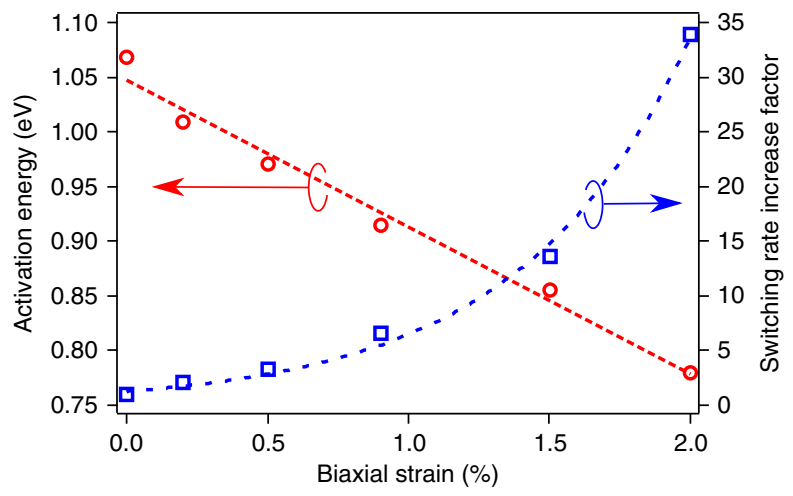

Figure 1 | The calculated activation energy for a single Ge atom to move from the GeTe layer into the van der Waals gap. (a) The 81 atom model at different stages of the atomic transition. The initial, intermediate saddle point and the final structures for the Ge atomic transition are shown, where $\mathrm{Ge}, \mathrm{Sb}$ and Te atoms are coloured black, blue and orange, respectively. The extent to which the atoms are shaded indicates depth in the simulation cell. The black box is the periodic boundary of the simulation cell. (b) The activation energy (red) for the transition is plot as function of biaxial strain. The increase in switching probability at $950 \mathrm{~K}$ relative to the unstrained superlattice is also plot as a function of biaxial strain (blue). 
Table 1 | Superlattice lattice parameters.

\begin{tabular}{|c|c|c|c|c|c|c|c|c|c|}
\hline \multicolumn{2}{|c|}{ Layer thickness $(\AA)$} & \multicolumn{4}{|c|}{ Lattice parameter $(\AA)$} & \multicolumn{4}{|c|}{ In-plane biaxial strain (\%) } \\
\hline \multirow{2}{*}{$\mathrm{b}_{2} \mathrm{Te}_{3}$} & \multirow[t]{2}{*}{ GeTe } & \multicolumn{2}{|c|}{ X-ray diffraction } & \multicolumn{2}{|c|}{ DFT } & \multicolumn{2}{|c|}{ X-ray diffraction } & \multicolumn{2}{|c|}{ DFT } \\
\hline & & a & c & $\mathbf{a}$ & c & $\mathrm{Sb}_{2} \mathrm{Te}_{3}$ & GeTe & $\mathrm{Sb}_{2} \mathrm{Te}_{3}$ & GeTe \\
\hline Sulk & 0 & 4.26 & 30.43 & 4.341 & 31.069 & 0.00 & NA & 0.00 & NA \\
\hline 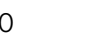 & Bulk & 4.16 & 10.66 & 4.237 & 10.887 & NA & 0.00 & NA & 0.00 \\
\hline 0 & 10 & 4.19 & 19.38 & 4.268 & 17.832 & -1.64 & 0.72 & -1.68 & 0.73 \\
\hline 20 & 10 & 4.22 & 30.12 & 4.269 & 28.522 & -0.94 & 1.44 & -1.66 & 0.76 \\
\hline 40 & 10 & 4.25 & 50.11 & 4.311 & 49.201 & -0.23 & 2.16 & -0.69 & 1.75 \\
\hline
\end{tabular}

DFT, density functional theory; NA, not applicable.

The $\mathrm{Sb}_{2} \mathrm{Te}_{3}-\mathrm{GeTe}$ superlattice lattice parameters for varying $\mathrm{Sb}_{2} \mathrm{Te}_{3}$ layer thicknesses are presented. These hexagonal lattice parameters were obtained by Rietveld fitting the $\mathrm{X}$-ray diffraction patterns shown in Supplementary Fig. 1 and Supplementary Note 1. The DFT modelled lattice parameters are also shown. These lattice parameters were used to calculate the measured and modelled in-plane biaxial strain for the $\mathrm{Sb}_{2} \mathrm{Te}_{3}$ and GeTe layers relative to their bulk values.

the $\mathrm{Sb}_{2} \mathrm{Te}_{3}-\mathrm{GeTe}$ superlattice is dominated by the vertical movement of $\mathrm{Ge}$ atoms through a plane of $\mathrm{Te}$ atoms. By calculating the activation energy for a single Ge atom to switch through the planar Te layer using the nudged elastic band transition state search method, which is illustrated in Fig. 1a, we find that the activation energy decreases linearly as the biaxial tensile strain is increased. Consequently the atomic switching probability at $950 \mathrm{~K}$ for the structure biaxially strained by $1.5 \%$ increases by an order of magnitude relative to the unstrained structure, see Fig. 1b. The calculated reduction in activation energy for atomic switching assumes that the $\mathrm{Sb}_{2} \mathrm{Te}_{3}$ and $\mathrm{GeTe}$ layers are stable but in reality a high temperature, close to the structure's melting point, is required for a significant proportion of the interfacial atoms to undergo atomic switching.

Applying strain to layers in a van der Waals superlattice. $\mathrm{Sb}_{2} \mathrm{Te}_{3}$ has a larger in-plane lattice constant than GeTe. To assess the tensile strain applied to the GeTe layers of $\mathrm{Sb}_{2} \mathrm{Te}_{3}-\mathrm{GeTe}$ superlattices, we grew different superlattice structures with the $\mathrm{Sb}_{2} \mathrm{Te}_{3}$ layer thickness varying from 1 to $4 \mathrm{~nm}$, whilst the GeTe layers' thickness was fixed at $1 \mathrm{~nm}$. The superlattice lattice parameters were measured by X-ray diffraction in a symmetric geometry. Only the (00L) peaks were present in the diffraction patterns thus indicating highly textured, layered superlattice films, see Supplementary Fig. 1 and Supplementary Note 1. The measured lattice parameters for GeTe in a hexagonal setting 13,22 were used to calculate the biaxial tensile strain in the $\mathrm{Sb}_{2} \mathrm{Te}_{3}$ and GeTe layers relative to their bulk values, see Supplementary Fig. 2. Table 1 shows that as the $\mathrm{Sb}_{2} \mathrm{Te}_{3}$ layer thickness is increased, the biaxial strain in the GeTe layer increases from 0.72 to $2.16 \%$. By comparing these measured strain values with the activation energy, which is shown in Fig. 1, it is clear that the activation energy for switching can be greatly reduced by increasing the thickness of the $\mathrm{Sb}_{2} \mathrm{Te}_{3}$ layers. Therefore a lower switching energy is to be expected for $\mathrm{Sb}_{2} \mathrm{Te}_{3}-\mathrm{GeTe}$ structures with thicker $\mathrm{Sb}_{2} \mathrm{Te}_{3}$ layers.

Premelt disordering in strained van der Waals superlattices. It is clear from Table 1 that when a $2 \mathrm{D} \mathrm{GeTe}$ crystal is incorporated in an $\mathrm{Sb}_{2} \mathrm{Te}_{3}-\mathrm{GeTe}$ superlattice heterostructure, the GeTe crystal is strained and therefore distorted with respect to the bulk GeTe crystal. We therefore investigated the effect of biaxial strain on the stability of the GeTe layers within the $\mathrm{Sb}_{2} \mathrm{Te}_{3}-\mathrm{GeTe}$ heterostructure by DF/MD simulations. The crystallinity of the individual GeTe and $\mathrm{Sb}_{2} \mathrm{Te}_{3}$ layers was calculated as a function of time. The crystallinity evolution of the individual $\mathrm{Sb}_{2} \mathrm{Te}_{3}$ and GeTe layers for the $2 \%$ biaxially strained superlattice is shown in Fig. 2a whilst the crystallinity plots for other strains are given in Supplementary Fig. 3 and discussed in Supplementary Note 2. a

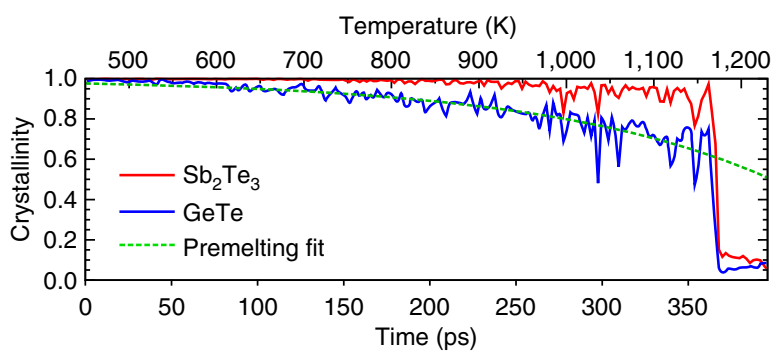

b

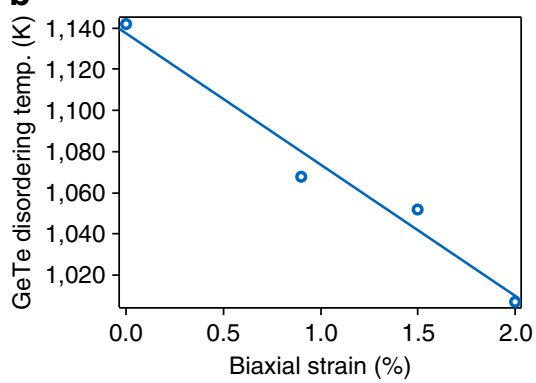

C

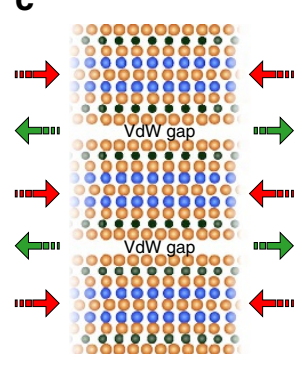

Figure 2 | Strain-controlled interfacial premelting. (a) The crystallinity evolution for the $2 \%$ biaxially strained $\mathrm{Sb}_{2} \mathrm{Te}_{3}-\mathrm{GeTe}$ vdW superlattice. The crystallinity of the $\mathrm{Sb}_{2} \mathrm{Te}_{3}$ layers (red) remains close to 1.0 until the superlattice structure melts. In contrast, the GeTe layers (blue) premelts at $\sim 160 \mathrm{~K}$ below the melting point of the $\mathrm{Sb}_{2} \mathrm{Te}_{3}$ layer. The dashed green line is a fit to the GeTe crystallinity and was used to determine the temperature that the crystallinity dropped to 0.8 , which we defined as the disordering temperature. Similar plots for $0 \%$ strain, 0.9 and $1.5 \%$ biaxial strain are given in Supplementary Fig. 3. (b) The disordering temperature of the GeTe layers is plot as a function of biaxial strain in the GeTe layers. Increasing the biaxial strain decreases the GeTe disordering temperature. (c) Relaxed $\mathrm{Sb}_{2} \mathrm{Te}_{3}-\mathrm{GeTe}$ vdW heterostructure. The direction of strain in the $2 \mathrm{D}$ crystal lattices alternates between tensile ( $\mathrm{GeTe}$ ) and compressive $\left(\mathrm{Sb}_{2} \mathrm{Te}_{3}\right)$ relative to their respective bulk crystal lattices. The red and green arrows indicate the layers subjected to compressive and tensile strain respectively. The atomic colours are: Ge atoms-black, Sb atoms-blue, and Te atoms-orange.

Both the $\mathrm{Sb}_{2} \mathrm{Te}_{3}$ and the GeTe layers show an abrupt decrease in crystallinity at a similar melting temperature. However, the GeTe crystallinity gradually decreases as the temperature increases above $600 \mathrm{~K}$. In effect, premelt disordering starts to occur in a $5 \AA$ thick GeTe 2D plane at a temperature $300 \mathrm{~K}$ below the melting temperature of the whole structure. Although melting is stochastic by nature, there is a clear trend that increasing biaxial strain decreases this GeTe premelt disordering temperature, as shown in Fig. 2b. Thus we conclude that disordering in the GeTe 
layers of the superlattice is highly sensitive to biaxial strain, which can be controlled by changing the $\mathrm{Sb}_{2} \mathrm{Te}_{3}$ layers' thickness.

Diffusive atomic switching in vdW superlattices. We used femtosecond laser pulses to measure the energy required to switch the superlattices into a low reflectivity state. Single $30 \mathrm{fs}$ laser pulses at a wavelength of $800 \mathrm{~nm}$ were used to switch $40 \mathrm{~nm}$ thick $\mathrm{Sb}_{2} \mathrm{Te}_{3}-\mathrm{GeTe}$ crystalline superlattice samples into a low reflectivity state. The minimum laser fluence required to write a low reflectivity spot into the film is shown as a function of strain in Fig. 3a. An example of the laser fluence measurement is shown in Supplementary Fig. 4. Increasing the biaxial strain in the GeTe layers by increasing the $\mathrm{Sb}_{2} \mathrm{Te}_{3}$ layer thickness reduces the fluence necessary for switching the optical properties of the structure. For reference, the measured switching energy for cubic $\mathrm{Ge}_{2} \mathrm{Sb}_{2} \mathrm{Te}_{5}$ is also shown. It is worth noting that the superlattice composed of $1 \mathrm{~nm}$ thick $\mathrm{Sb}_{2} \mathrm{Te}_{3}$ layers and $1 \mathrm{~nm}$ thick GeTe layers has the average composition $\mathrm{Ge}_{2} \mathrm{Sb}_{2} \mathrm{Te}_{5}$. However, its threshold switching energy is lower than the $\mathrm{Ge}_{2} \mathrm{Sb}_{2} \mathrm{Te}_{5}$ alloy, which shows that the superlattice structure can be used to design and optimize switching in $\mathrm{Sb}_{2} \mathrm{Te}_{3}-\mathrm{GeTe}$-based materials. Moreover, the $\mathrm{GeTe}$ layers in superlattices composed of $1 \mathrm{~nm}$ thick $\mathrm{Sb}_{2} \mathrm{Te}_{3}$ layers and a

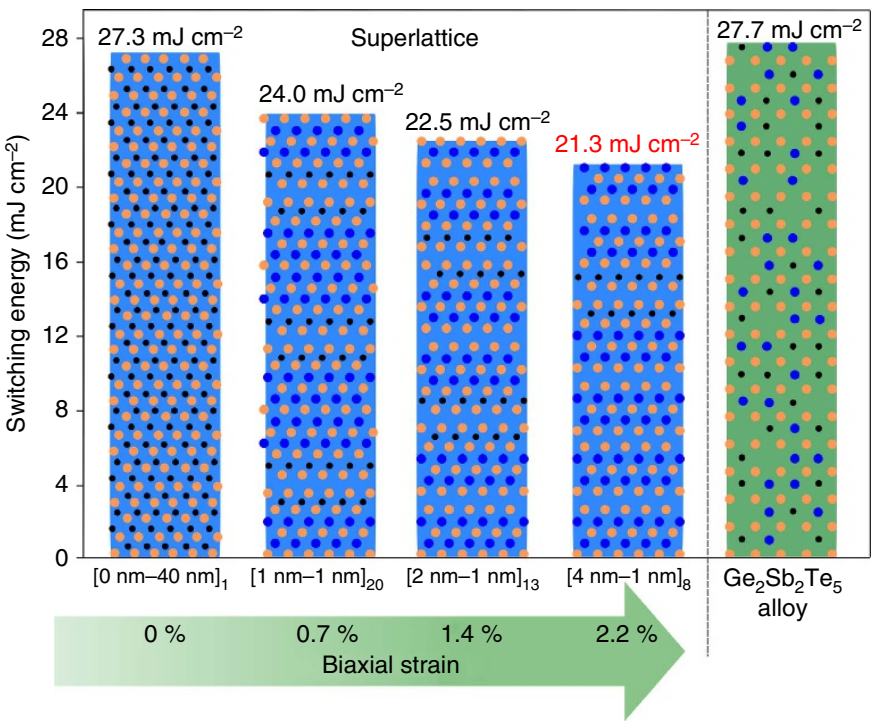

b

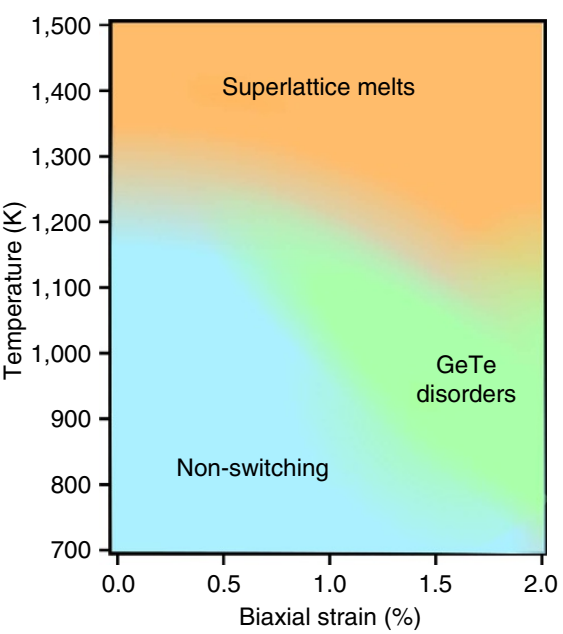

c

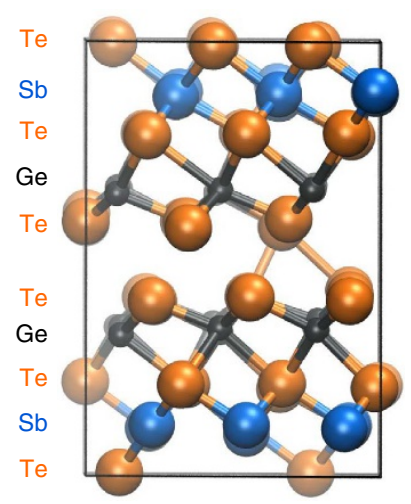

d

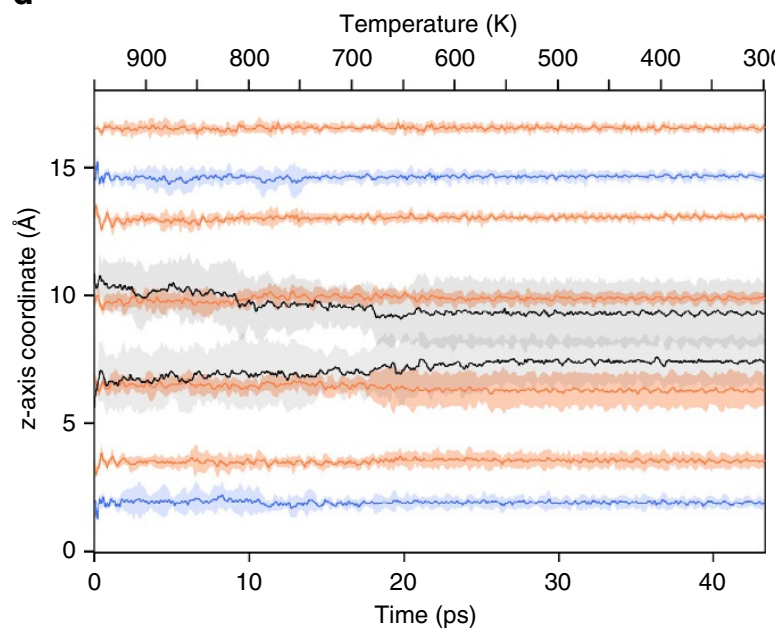

e

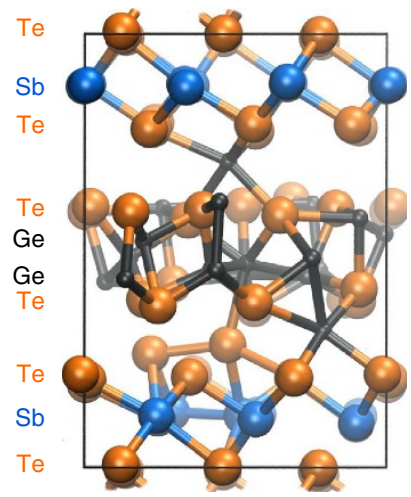

Figure 3 | GeTe disordering. (a) The measured minimum laser fluence required to decrease the optical reflectivity of the $\mathrm{Sb}_{2} \mathrm{Te}_{3}-\mathrm{GeTe}_{\mathrm{vdW}} \mathrm{superlattices}$ is shown for different thicknesses of the $\mathrm{Sb}_{2} \mathrm{Te}_{3}$ layers (blue coloured bars). The biaxial strain in the GeTe layers increases with the $\mathrm{Sb}_{2} \mathrm{Te}_{3}$ layer thickness, see Table 1, resulting in a lower threshold fluence to induce disordering in the GeTe layers. The switching fluence required for the $\mathrm{Ge}_{2} \mathrm{Sb}_{2} \mathrm{Te}_{5}$ alloy is also shown (green coloured bar). The dots on the bars illustrate the atomic layer sequence where the $\mathrm{Ge}, \mathrm{Sb}$, and Te atoms are coloured black, blue, and orange respectively. (b) A strain-temperature map to show the regions where the GeTe and SbTe layers remain stable (blue area), and where diffusive Ge atomic disordering (green area) and superlattice melting (orange area) are likely. GeTe disordering is possible when the superlattice structure is strained. (c) The initial $\left(\mathrm{Sb}_{2} \mathrm{Te}_{3}, 1 \mathrm{~nm}-\mathrm{GeTe}, 1 \mathrm{~nm}\right)$ superlattice structure in the resonantly bonded crystalline state. (d) The mean $z$-coordinate evolution of the atoms in each layer during a simulated quench from $950 \mathrm{~K}$ for the 1.5\% strained superlattice structure (two similar quenches from 950 and $1,400 \mathrm{~K}$ for the $0 \%$ strained superlattice are included in Supplementary Figs 7 and 8, and supporting information is given in Supplementary Note 4). The solid lines show the mean position of the nine atoms in each layer where Ge, Sb and Te atoms are coloured black, blue and orange, respectively. The semitransparent shading indicates the standard deviation, $\pm \sigma$, in the $z$-coordinate and was calculated according to $\sigma=\sqrt{\frac{1}{8} \sum_{i=1}^{9}\left(z_{i}-\bar{z}\right)^{2}}$, where $\bar{z}$ is the mean $z$-coordinate of the nine atoms in each layer and $i$ is the atom index in the layer. (e) The structure after quenching to $300 \mathrm{~K}$. The $\mathrm{Sb}_{2} \mathrm{Te}_{3}$ layers remain in tack after the quench, whilst diffusive atomic disordering is confined to a $5 \AA$ thick two-dimensional layer of GeTe layer. Colours: Ge-black, Sb-blue and Te-orange. 
$1 \mathrm{~nm}$ thick GeTe layers are subjected to almost $1 \%$ biaxial strain relative to the unstrained $40 \mathrm{~nm}$ thick GeTe film, see Table 1 . We see in Fig. 3a that this also substantially reduces the threshold switching energy relative to the crystalline GeTe film.

To confirm the reversibility of the switching process the optical reflectivity of the laser switched marks was measured before and after annealing the samples at $220^{\circ} \mathrm{C}$. The optical reflectivity for all the samples typically increased by $15 \%$ after annealing such that the switched areas were indistinguishable from the background crystalline film. This indicates the reversibility of the GeTe disordering process (Supplementary Figs 5 and 6).

To establish the influence of strain and temperature on the relative ability for the GeTe layer to diffusively disorder, we used $\mathrm{DF} / \mathrm{MD}$ to map the strain-temperature parameter space and find the conditions required for $\mathrm{Ge}$ atoms to diffuse into the vdW gap. Recent time resolved structural measurements of femtosecond laser amorphisation of crystalline $\mathrm{Ge}_{2} \mathrm{Sb}_{2} \mathrm{Te}_{5}$ showed that the melting process is typically complete in 5 ps (ref. 12). Therefore for different levels of biaxial strain we estimated the required temperature to switch at least one Ge atom into the vdW gap in 5 ps. The map shown in Fig. $3 b$, which is detailed in Supplementary Table 1 and Supplementary Note 3, shows that the unstrained structure is stable up to $1,300 \mathrm{~K}$ and $\mathrm{Ge}$ atomic switching is unlikely during the $5 \mathrm{ps}$ simulation. When the temperature is raised to $1,400 \mathrm{~K}$, the $\mathrm{Sb}_{2} \mathrm{Te}_{3}$ and GeTe layers melt and inter-diffuse. However, we find that biaxial tensile strain greatly enhances $\mathrm{Ge}-\mathrm{Te}$ atomic switching by premelting the $5 \AA$ thick GeTe layer. When the $\mathrm{Sb}_{2} \mathrm{Te}_{3}-\mathrm{GeTe}$ heterostructure is biaxially strained by $0.9 \%, \mathrm{Ge}-\mathrm{Te}$ diffusive atomic switching occurs at temperatures below the superlattice melting temperature. Further increases in tensile biaxial strain widens the temperature window for diffusive atomic switching.

The stability of the switched phase was studied with long quench runs where the structure was heated to temperatures ranging from 700 to $1,400 \mathrm{~K}$, and cooled to $300 \mathrm{~K}$ at a rate of $-15 \mathrm{~K} \mathrm{ps}^{-1}$ to mimic the effect of a short laser heating pulse and subsequent thermal dissipation. Diffusive $\mathrm{Ge}-\mathrm{Te}$ atomic switching can be observed by plotting the $z$-coordinate of the atoms in the simulation cell as a function of time and temperature, see Fig. 3d. Ge-Te atomic switching is visualized as an exchange of the $\mathrm{Ge}$ and $\mathrm{Te}$ relative layer positions and a crossing of their $z$-coordinates in the evolution plot. If the structure melts, the atomic layers inter-diffuse and the resultant plot does not show the regular $z$-coordinate spacing that is observed for a layered solid. Examples of $z$-coordinate evolution plots for $0 \%$ strain that are quenched from different temperatures are given in Supplementary Figs 7 and 8, and described in Supplementary Note 4.

Figure $3 \mathrm{c}-\mathrm{e}$ shows the structural evolution of the $\mathrm{Sb}_{2} \mathrm{Te}_{3}-\mathrm{GeTe}$ heterostructure that was subjected to $1.5 \%$ biaxial strain and an initial temperature of $950 \mathrm{~K}$. After $\sim 10$ ps most of the Ge atoms have moved into the Te-Te vdW gap producing disordered $2 \mathrm{D}$ GeTe layers encapsulated by crystalline $\mathrm{Sb}_{2} \mathrm{Te}_{3}$ quintuple blocks. The bottom layer Ge atoms completely switch into the gap within approximately 1 ps, however this is likely to be an artefact of the molecular dynamics initial conditions, and therefore we focus on the movement at $10 \mathrm{ps}$ where the system has had some time to equilibrate. By quenching the structure at $-15 \mathrm{Kps}^{-1}$, the switched Ge atoms are frozen between the Te-Te layers and this is visible as a dampening of the atomic movement about their equilibrium $z$-position. The structure retains a layered nature after the diffusive switching.

\section{Discussion}

We have shown that the activation energy for Ge atoms to diffuse into the vdW gap (Fig. 1b), the temperature for premelt disordering of the GeTe layers (Fig. 2b), and the switching energy required to observe a reduction in optical reflectivity (Fig. 3a) are all lowered by biaxially straining the superlattice GeTe layers. Applying biaxial tensile strain to the $\mathrm{Sb}_{2} \mathrm{Te}_{3}-\mathrm{GeTe}$ interface increases the planar separation of $\mathrm{Te}$ atoms and reduces the energy barrier for the Ge atoms to move through the Te plane, and this explains the measured lower switching energy.

In practice it is common to strain crystalline films by heteroepitaxial growth from a substrate with a small lattice mismatch, or by capping with a stressed film. However, these techniques do not apply to vdW bonded structures because the in-plane lattice constants of vdW solids usually relax to the bulk values after three vdW interfaces ${ }^{23}$. However, we use an $\mathrm{Sb}_{2} \mathrm{Te}_{3}-\mathrm{GeTe}$ superlattice with $\mathrm{Sb}_{2} \mathrm{Te}_{3}$ layers less than four quintuple blocks thick. This means the GeTe layers reset the strain in the $\mathrm{Sb}_{2} \mathrm{Te}_{3}$ layers before it can fully relax. Since the $a$ lattice parameter of the bulk $\mathrm{Sb}_{2} \mathrm{Te}_{3}$ crystal is $2.4 \%$ larger than that of bulk GeTe, it is possible to biaxially strain the GeTe layer by increasing the thickness of the $\mathrm{Sb}_{2} \mathrm{Te}_{3}$ layers within the superlattice. We find that the superlattice takes a weighted average in-plane lattice parameter of the GeTe and $\mathrm{Sb}_{2} \mathrm{Te}_{3}$ bulk crystal lattice parameters.

Ideally, as the strain applied to the sample increases, the $\mathrm{Sb}_{2} \mathrm{Te}_{3}$ scaffold layers should remain stable whilst the GeTe layers should become increasingly unstable, which is necessary for diffusive atomic switching. This requires a bond hierarchy to exist within the heterostructure. Crystalline $\mathrm{GeTe}$ and related phase change materials, such as $\mathrm{Ge}_{2} \mathrm{Sb}_{2} \mathrm{Te}_{5}$, have an extraordinarily large optical refractive index compared with that of their amorphous structure. This is due to the existence of highly polarizable $p$-orbital electrons in crystalline GeTe and less polarizable bonds in the amorphous state ${ }^{24}$. The GeTe crystal is characterized by a rhombohedral unit cell with the Ge atom lying off-centre along the [111] direction to give three long, and three short $\mathrm{Ge}-\mathrm{Te}$ bonds, and therefore a bond energy hierarchy exists for this crystal. DFT calculations have shown that electrons are delocalized across multiple unit cells along the longer $\mathrm{Ge}-\mathrm{Te}$ bonds ${ }^{25}$, which explains its high polarizability and concomitantly large refractive index. This type of delocalized bonding is commonly referred as 'resonance bonding' and was introduced in the context of IV-VI compounds, including GeTe, by Lucovsky and White ${ }^{26}$. In contrast, the shorter GeTe bonds are localized at the mid-point between the $\mathrm{Ge}$ and $\mathrm{Te}$ atoms. The stability of GeTe is dependent on the rather fragile alignment of resonant bonds across multiple unit cells; hence the GeTe crystal is particularly sensitive to structural distortions ${ }^{25}$.

The GeTe premelt disordering, which is shown in Fig. 2a,b, at a temperature below the melting temperature of $\mathrm{Sb}_{2} \mathrm{Te}_{3}$ may seem surprising when considering the higher bulk melting temperature of GeTe $(997 \mathrm{~K})^{27}$, compared with $\mathrm{Sb}_{2} \mathrm{Te}_{3}(891 \mathrm{~K})^{28}$. However, we highlight again the sensitivity of the GeTe crystal to distortions ${ }^{25}$. For small distortions, such as those encountered by straining the GeTe lattice, resonance bonding is weakened but not broken. We see from Table 1 that as the thickness of the $\mathrm{Sb}_{2} \mathrm{Te}_{3}$ layer is increased, the GeTe lattice is put under biaxial tensile strain and consequently the Ge-Te resonant bonds are weakened and the disordering temperature of the GeTe layers is lowered. In contrast, the lattice parameter of the $\mathrm{Sb}_{2} \mathrm{Te}_{3}$ layers tend to the bulk value and therefore the layers remain crystalline, see Fig. $2 \mathrm{a}$ and Supplementary Fig. 3. In effect, the unstrained superlattice structure consists of biaxially compressed $\mathrm{Sb}_{2} \mathrm{Te}_{3}$ and biaxially tensile strained GeTe, which effectively increases the stability of the $\mathrm{Sb}_{2} \mathrm{Te}_{3}$ layer whilst decreasing the stability of the GeTe layer. This situation is illustrated in Fig. 2c. Consequently, increasing the $\mathrm{Sb}_{2} \mathrm{Te}_{3}$ thickness increases the strain in the GeTe layer, destabilizing it and promoting premelt disordering. 
The reduction in the laser energy used to disorder the GeTe layers, which is shown in Fig. 3a, is due to strain induced destablization of resonant bonds. This results in preferential disordering of GeTe layers at temperatures below the superlattice melting temperature. The disordered GeTe layers produce a superlattice structure with a lower optical reflectivity. This is to be expected since disorder forbids resonant bonding, which is responsible for the extraordinarily large refractive index of the GeTe crystal. The high reflectivity, switched, state was readily recovered for all structures after annealing the samples at $220^{\circ} \mathrm{C}$. Supplementary Figs 6 and 9 show that both the measured and modelled reflectivity modulation is $\sim 10 \%$ in the visible spectrum (further details are given in Supplementary Notes 5 and 6). It is worth noting that phase change memory cells composed of similar superlattice structures exhibit excellent cycleability ${ }^{13}$ and we expect that these strained superlattices should also be highly cycleable between the high and low reflectivity states.

Figure $3 \mathrm{c}-\mathrm{e}$ shows that the $\mathrm{Sb}_{2} \mathrm{Te}_{3}$ layers can remain crystalline during the GeTe disordering. During the 43 ps simulation $\mathrm{Sb}_{2} \mathrm{Te}_{3}$ atoms retained their crystallographic positions. Disordering is confined to the GeTe layers that are less than $5 \AA$ thick-a 2D phase transition occurs. The final 'switched' structure is shown in Fig. 3e. While the activation energy for a single $\mathrm{Ge}$ atom penetrating the Te plane is lowered by applying tensile strain, see Fig. 1b, we now also see a tendency for the GeTe layer to disorder after the transition. Note that this switching behaviour is in stark contrast to the GeTe layer in the unstrained structure at $950 \mathrm{~K}$, which does not disorder and all atoms remain in their initial unswitched crystallographic positions. At higher temperatures the $\mathrm{Sb}_{2} \mathrm{Te}_{3}$ layers in the unstrained superlattice are unstable and both the GeTe and $\mathrm{Sb}_{2} \mathrm{Te}_{3}$ layers inter-diffuse, see Supplementary Figs 7 and 8 .

From these results we conclude that diffusive $\mathrm{Ge}-\mathrm{Te}$ atomic switching in $\mathrm{Sb}_{2} \mathrm{Te}_{3}-\mathrm{GeTe}$ vdW heterostructures can be thermally stimulated by premelting the GeTe layer at temperatures lower than the melting point of $\mathrm{Sb}_{2} \mathrm{Te}_{3}$. Increasing the thickness of the $\mathrm{Sb}_{2} \mathrm{Te}_{3}$ layer increases the biaxial strain in the GeTe layer and causes a related increase in the atomic switching probability and a decrease in the switching energy. The $\mathrm{Sb}_{2} \mathrm{Te}_{3}$ layers experience little distortion during the switch and their rigidity is crucial for the stability of the whole superlattice structure. As such, $\mathrm{Sb}_{2} \mathrm{Te}_{3}$ presents a versatile scaffold to strain engineer the properties of GeTe. We conclude that in-plane biaxial strain significantly enhances diffusive atomic disordering within the GeTe layers of $\mathrm{Sb}_{2} \mathrm{Te}_{3}-\mathrm{GeTe}$ superlattices.

Herein, diffusive atomic switches based on strain-engineered $\mathrm{Sb}_{2} \mathrm{Te}_{3}-\mathrm{GeTe}$ heterostructures have been developed. However, we believe that the applicability of strain engineering can be generalized and extended to other vdW bonded heterostructures of $2 \mathrm{D}$ chalcogenide crystals and therefore we highlight the following rules that can be used to design similar diffusive atomic switching heterostructures: First, the switching material $(\mathrm{GeTe})$ in the heterostructure must exhibit resonant bonding such that its structure is sensitive to distortions and strain can be used to modify the disordering energy. Second, the scaffold material $\left(\mathrm{Sb}_{2} \mathrm{Te}_{3}\right)$ in the heterostructure must be stable over the temperature range that the switching material exhibits premelting. Third, the scaffold material must have a larger bulk lattice parameter, $a$, than the switching material. Fourth, it must be possible to grow the vdW heterostructure superlattices from $2 \mathrm{D}$ crystals of the switching and scaffold materials by vdW heteroepitaxy such that the layers strain one another.

The first point prevents these design rules being applied to phase change materials with tetrahedral crystal structures, such as $\mathrm{Cu}_{2} \mathrm{GeTe}_{3}$ (ref. 29) and $\mathrm{GaSb}$ (ref. 30), where resonant bond alignment across multiple unit cells is not possible. The third point is necessary such that the switching material is under tensile strain relative to its bulk, and therefore exhibits a melting temperature that is controlled by the thickness of the scaffold layers.

The ability to tune the strain profile of vdW heterostructures opens a further degree of freedom to manipulate their function and properties. We have demonstrated that diffusive disordering and atomic switching can occur by selectively destabilizing $5 \AA$ thick GeTe 2D crystal layers within a $\mathrm{Sb}_{2} \mathrm{Te}_{3}-\mathrm{GeTe}$ vdW heterostructure superlattice. The extent of the destablization is controlled by the thickness of the $\mathrm{Sb}_{2} \mathrm{Te}_{3}$ layers, which strain the $\mathrm{GeTe}$ layers. Consequently, thicker $\mathrm{Sb}_{2} \mathrm{Te}_{3}$ layers cause a significant decrease in the energy necessary for switching the properties of $\mathrm{Sb}_{2} \mathrm{Te}_{3}-\mathrm{GeTe}$ superlattices. More generally, four rules have been identified that can be used to aid the design of $2 \mathrm{D}$ crystal vdW heterostructures with switchable properties.

\section{Methods}

Computational methods. All DF/MD runs were performed using the Vienna Ab initio Simulation Package (VASP 5.3.3) ${ }^{31}$ with PAW-pseudopotentials ${ }^{32}$, $\mathrm{PBE}$ exchange-correlation functional ${ }^{33}, 3 \mathrm{fs}$ timestep, and an NVT ensemble with periodic boundary conditions. For the superlattice structure geometry optimizations at $0 \mathrm{~K}$, which were used to calculate the superlattice lattice parameters given in Table 1 , we used a hexagonal unit cell with a $\Gamma$ centred mesh of $8 \times 8 \times 2$ points. We used a plane-wave basis with $240 \mathrm{eV}$ cutoff. The energy precision for the self consistent field calculation was set to $10^{-6} \mathrm{eV}$.

To model the effect of biaxial strain on diffusive atomic transitions, an 81 atom $\mathrm{Sb}_{2} \mathrm{Te}_{3}-\mathrm{GeTe}$ model was built. For the DF/MD runs energies were calculated at the $\Gamma$ point of the Brillouin zone $(\mathbf{k}=0)$, which led to an accuracy of $50 \mathrm{meV}$ per atom relative to a $5 \times 5 \times 5 \mathrm{k}$-point mesh. We used a plane-wave basis with $220 \mathrm{eV}$ cutoff. The energy precision for the self consistent field calculation was set to $10^{-5} \mathrm{eV}$. The temperature of the model was controlled by velocity rescaling. The superlattice unit cell dimensions were estimated by running $10 \mathrm{ps}$ of MD at $300 \mathrm{~K}$ with different cell dimensions. The $300 \mathrm{~K}$ relaxed cell dimensions were then found by selecting the structure with the lowest square-average stress tensor diagonal elements over the last 5 ps of the trajectory. This cell was then elongated by the desired strain amount in the $x y$-plane, and similar 10 ps simulations were performed to find the relaxed $z$-length while keeping the lateral strain.

Crystallinity was measured with bond orientational (BO) order parameter of Steinhardt et al. ${ }^{34}$, which was also used previously in crystallization simulations of $\mathrm{Ge}_{2} \mathrm{Sb}_{2} \mathrm{Te}_{5}$ (ref. 35). The $\mathrm{BO}$ order parameter was calculated by projecting the bond vectors onto a basis of spherical harmonics $Y_{l m}$, with a suitable $l$ value. The first nonzero $l$ value for cubic lattice, $l=4$ was used. The order parameter $\mathbf{Q}_{l}$ is defined as

$$
\mathbf{Q}_{l}(i)=\sqrt{\frac{4 \pi}{2 l+1} \sum_{m=-l}^{l}\left|\frac{1}{N_{\mathrm{b}}(i)} \sum_{k=0}^{N_{\mathrm{b}}(i)}\left(\frac{1}{N(i)} \sum_{j=1}^{N(i)} Y_{l m}\left(\mathbf{r}_{i j}\right)\right)\right|^{2}}
$$

where $\mathbf{r}_{i j}$ is the vector between atoms $i$ and $j, N(i)$ is the number of neighbours for atom $i$, and $N_{\mathrm{b}}$ includes the atom $i$ and its neighbours. The $\mathbf{Q}_{4}(i)$ value for ideal, vacancy-free rock-salt lattice is 0.764 . To compensate for thermal fluctuations we defined a crystalline atom as having a $\mathbf{Q}_{4}(i)$ magnitude greater than 0.6 . This method results in a good agreement with the atoms marked as crystalline by the order parameter and the atoms that look crystalline in visualizations.

The premelting in the $\mathrm{Sb}_{2} \mathrm{Te}_{3}-\mathrm{GeTe}$ superlattice was studied with a temperature ramp for each strain. The initial temperature was $450 \mathrm{~K}$, and it was increased at $1.97 \mathrm{~K} \mathrm{ps}^{-1}$ until the structure melted. The initial velocities were random, and scaled according to the temperature. The layer crystallinities were calculated as an average over all the atoms occupying the space initially occupied by each layer, and over 1 ps $(1.97 \mathrm{~K})$ windows.

The activation energy for a single Ge atom to switch into the vdW gap was calculated with the DFT code CASTEP ${ }^{36}$ using the 81 atom $\mathrm{GeTe}-\mathrm{Sb}_{2} \mathrm{Te}_{3}$ model cell with ultra-soft pseudo-potentials. The plane-wave basis cutoff energy was set to $230 \mathrm{eV}$. The atomic positions and the cell shape was relaxed to the ground state using the $\mathrm{BFGS}^{37}$ minimizer with an energy tolerance of $5 \times 10^{-6} \mathrm{eV}$ per atom. The maximum force on the atoms was less than $0.01 \mathrm{eV}^{-1}$. The energy was calculated at the gamma point. The atomic positions of the switched structure were also relaxed using the same criteria as listed above. The simulation cell volume was fixed with the non-switched volume of $2,501.63 \AA^{3}$. The nudged elastic band method was then used to calculate the activation energy for a single Ge atom to switch into the vdW gap. A linear synchronous transit (LST) search was used to initially approximate the barrier height. The activation energy was then refined by cyclically repeating a quadratic synchronous transit (QST) maximization with a conjugate gradient minimization until the stationary point of the barrier was found ${ }^{38}$. The relative probability that the Ge atoms moved into the vdW gap was 
calculated using the Arrhenius equation $k=A e^{-\frac{E_{A}}{k_{\mathrm{B}} T}}$, as a function of strain at $950 \mathrm{~K}$.

Note that the DFT energy of our models was not corrected for the inter-block $\mathrm{vdW}$ interaction. Our strain-engineered switching model is based on the GeTe layer disordering process being dominated by the in-plane separation of $\mathrm{Te}-\mathrm{Te}$ bonds rather than the out-of-plane vdW interactions. Table 1 shows that the error between the DFT modelled in-plane lattice parameter and the X-ray diffraction measured in-plane lattice parameter is $<2 \%$. Therefore the PBE exchangecorrelation functional has use for modelling biaxially strained layered materials. Indeed, our simple 81 atom model proved to be an essential tool for designing superlattice structures with in-plane biaxial strain. Extending our models into a full simulation would involve accounting for $\mathrm{vdW}$ interactions, and fully simulating the $\left(\mathrm{Sb}_{2} \mathrm{Te}_{3}, 2 \mathrm{~nm}-\mathrm{GeTe}, 1 \mathrm{~nm}\right)$ and $\left(\mathrm{Sb}_{2} \mathrm{Te}_{3}, 4 \mathrm{~nm}-\mathrm{GeTe}, 1 \mathrm{~nm}\right)$ superlattice structures to find their in-plane lattice parameter, rather than modelling the larger lattice parameter by straining the $\left(\mathrm{Sb}_{2} \mathrm{Te}_{3}, 1 \mathrm{~nm}-\mathrm{GeTe}, 1 \mathrm{~nm}\right)$ structure, which we did here.

$\mathbf{S b}_{\mathbf{2}} \mathrm{Te}_{\mathbf{3}}$-GeTe superlattice growth. The $\left(\mathrm{Sb}_{2} \mathrm{Te}_{3}, x \mathrm{~nm}-\mathrm{GeTe}, 1 \mathrm{~nm}\right)$, where $x=1,2$ and 4 , superlattice films for laser switching measurements were grown on Si (100) substrates using layer-by-layer sputter deposition. Before the superlattice deposition the native oxide was removed from the $\mathrm{Si}$ substrate using a $30 \mathrm{~W}$ RF plasma for $60 \mathrm{~min}$ at a substrate holder temperature of $300^{\circ} \mathrm{C}$. A 10 -nm thick $\mathrm{Sb}_{2} \mathrm{Te}_{3}$ buffer layer was then grown on the Si surface before the deposition of the superlattice film. The samples were grown in an Ar atmosphere of $0.5 \mathrm{~Pa}$, whilst the base pressure of the vacuum chamber was better than $2.5 \times 10^{-5} \mathrm{~Pa}$. The substrate holder temperature was held at $300^{\circ} \mathrm{C}$ during the superlattice growth. For all samples the total film thickness was kept constant at $40 \mathrm{~nm}$ and the composition was confirmed by energy dispersive X-ray (EDX) spectroscopy. The lattice parameters $\left(a_{\mathrm{SL}}, c_{\mathrm{SL}}\right)$ of the superlattices were determined by Rietveld refinement. The in-plane biaxial strain applied to the GeTe and $\mathrm{Sb}_{2} \mathrm{Te}_{3}$ layers is dictated by the superlattice in-plane lattice parameters, and this was estimated by $\epsilon_{\mathrm{GeTe}}=\frac{a_{\mathrm{SL}}-a_{\mathrm{GT}}}{a_{\mathrm{GT}}}$

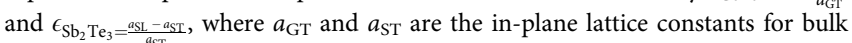
GeTe and bulk $\mathrm{Sb}_{2} \mathrm{Te}_{3}$ in a hexagonal setting. To avoid encapsulation induced stresses ${ }^{39}$ all films were uncapped. To minimize oxidation the samples were either measured immediately after growth or stored under vacuum until the measurement was conducted.

Switching of superlattice samples. Samples were mounted on a linear XY stage with the surface normal to the incident laser beam. The laser was focused to a Gaussian spot with a radius of $260 \mu \mathrm{m}$. A Pockels cell was used to extract a single femtosecond laser pulse on demand and the pulse energy was controlled by a half wave plate and polariser based attenuator. After each laser pulse, the sample was moved to a new position. The sample was then examined in a microscope to observe the phase transformation. Below a critical fluence $F_{\mathrm{A}}$ the laser did not induce a permanent change in the reflectivity. Above $F_{\mathrm{A}}$, the contrast between crystalline and amorphous phases grew until the contrast saturated and the lateral size of the switched area increased (sample images are provided in Supplementary Fig. 5). Further increasing the fluence eventually resulted in ablation and damage to the film. For further details see Supplementary Note 5.

Data availability. The data that support the findings of this study are available from the corresponding author upon request.

\section{References}

1. Geim, A. K. \& Grigorieva, I. V. Van der Waals heterostructures. Nature 499, 419-425 (2013).

2. Woods, C. R. et al. Commensurate-incommensurate transition in graphene on hexagonal boron nitride. Nat. Phys. 10, 451-456 (2014).

3. van der Zande, A. M. et al. Tailoring the electronic structure in bilayer molybdenum disulfide via interlayer twist. Nano. Lett. 14, 3869-3875 (2014).

4. Qian, X., Liu, J., Fu, L. \& Li, J. Quantum spin hall effect in two-dimensional transition metal dichalcogenides. Science 346, 1344-1347 (2014).

5. Takagaki, Y., Saito, Y. \& Tominaga, J. Manipulation of the presence of helical surface states of topological insulators using $\mathrm{Sb}_{2} \mathrm{Te}_{3}-\mathrm{GeTe}$ superlattices. App. Phys. Lett. 108, 112102 (2016)

6. Lee, M. \& Fitzgerald, E. Strained Si, SiGe, and Ge channels for high-mobility metaloxide-semiconductor field-effect transistors. J. Appl. Phys. 97, 011101 (2005).

7. Maiti, C. K. \& Maiti, T. K. Strain Engineered MOSFETS 1st edn (CRC Press, 2013).

8. Lee, C., Wei, X., Kysar, J. W. \& Hone, J. Measurement of the elastic properties and intrinsic strength of monolayer graphene. Science 321, 385-388 (2008).

9. Bertolazzi, S., Brivio, J. \& Kis, A. Stretching and breaking of ultrathin $\mathrm{MoS}_{2}$. ACS Nano 5, 9703-9709 (2011).

10. Li, J., Shan, Z. \& Ma, E. Elastic strain engineering for unprecedented materials properties. MRS Bull. 39, 108-114 (2014).

11. Fons, P. et al. Photoassisted amorphization of the phase-change memory alloy $\mathrm{Ge}_{2} \mathrm{Sb}_{2} \mathrm{Te}_{5}$. Phys. Rev. B 82, 041203 (2010).
12. Waldecker, L. et al. Time-domain separation of optical properties from structural transitions in resonantly bonded materials. Nat. Mater. 14, 991-995 (2015).

13. Simpson, R. E. et al. Interfacial phase-change memory. Nat. Nanotechnol. 6, 501-505 (2011).

14. Tominaga, J., Simpson, R., Fons, P. \& Kolobov, A. in Proceedings of the European Phase Change and Ovonic Science Symposium, 54-59 (2010).

15. Chong, T. et al. Phase change random access memory cell with superlattice-like structure. Appl. Phys. Lett. 88, 122114 (2006).

16. Tominaga, J. et al. Role of Ge switch in phase transition: Approach using atomically controlled $\mathrm{GeTe} / \mathrm{Sb}_{2} \mathrm{Te}_{3}$ superlattice. Jpn. J. Appl. Phys. 47, 5763-5766 (2008).

17. Wang, W. et al. Enabling universal memory by overcoming the contradictory speed and stability nature of phase-change materials. Sci. Rep. 2, 360 (2012).

18. Soeya, S., Shintani, T., Odaka, T., Kondou, R. \& Tominaga, J. Ultra-low switching power, crystallographic analysis, and switching mechanism for $\mathrm{Sn}_{X} \mathrm{Te}_{100-X} / \mathrm{Sb}_{2} \mathrm{Te}_{3}$ diluted superlattice system. App. Phys. Lett. 103, 053103 (2013).

19. Saito, Y., Tominaga, J., Fons, P., Kolobov, A. \& Nakano, T. Ab-initio calculations and structural studies of $(\mathrm{SiTe})_{2}\left(\mathrm{Sb}_{2} \mathrm{Te}_{3}\right)_{n}$ (n: 1, 2, 4 and 6) phase-change superlattice films. Phys. Status Solidi 8, 302-306 (2014).

20. Takaura, N. et al. in Electron Devices Meeting (IEDM) 29.2.1-29.2.4 (IEEE International, 2014).

21. Yu, X. \& Robertson, J. Modeling of switching mechanism in gesbte chalcogenide superlattices. Sci. Rep. 5, 12612 (2015).

22. Simpson, R. E., Fons, P., Kolobov, A. V., Krbal, M. \& Tominaga, J. Enhanced crystallization of $\mathrm{GeTe}$ from an $\mathrm{Sb}_{2} \mathrm{Te}_{3}$ template. App. Phys. Lett. 100, 021911 (2012).

23. Dang, W., Peng, H., Li, H., Wang, P. \& Liu, Z. Epitaxial heterostructures of ultrathin topological insulator nanoplate and graphene. Nano Lett. 10, 2870-2876 (2010).

24. Lencer, D., Salinga, M. \& Wuttig, M. Design rules for phase-change materials in data storage applications. Adv. Mater. 23, 2030-2058 (2011).

25. Kolobov, A. V., Krbal, M., Fons, P., Tominaga, J. \& Uruga, T. Distortiontriggered loss of long-range order in solids with bonding energy hierarchy. Nat. Chem. 3, 311-316 (2011).

26. Lucovsky, G. \& White, R. Effects of resonance bonding on the properties of crystalline and amorphous semiconductors. Phys. Rev. B 8, 660-667 (1973).

27. Balde, L., Legendre, B. \& Balkhi, A. Etude du diagramme d'equilibre entre phases du systeme ternaire germanium-etain-tellure. J. Alloys Compd. 216, 285-293 (1995).

28. Ghosh, G. The sb-te (antimony-tellurium) system. J. Phase Equilib. 15, 349-360 (1994).

29. Skelton, J., Kobayashi, K., Sutou, Y. \& Elliott, S. Origin of the unusual reflectance and density contrasts in the phase-change material $\mathrm{Cu}_{2} \mathrm{GeTe}_{3}$. App. Phys. Lett. 102, 224105 (2013).

30. Putero, M. et al. Phase transition in stoichiometric GaSb thin films: anomalous density change and phase segregation. Appl. Phys. Lett. 103, 231912 (2013).

31. Kresse, G. \& Hafner, J. Ab initio molecular dynamics for liquid metals. Phys. Rev. B 47, 558-561 (1993).

32. Kresse, G. From ultrasoft pseudopotentials to the projector augmented-wave method. Phys. Rev. B 59, 1758-1775 (1999).

33. Perdew, J., Burke, K. \& Ernzerhof, M. Generalized gradient approximation made simple. Phys. Rev. Lett. 77, 3865-3868 (1996).

34. Steinhardt, P. J., Nelson, D. R. \& Ronchetti, M. Bond-orientational order in liquids and glasses. Phys. Rev. B 28, 784 (1983).

35. Kalikka, J., Akola, J., Jones, R. O., Kohara, S. \& Usuki, T. Amorphous $\mathrm{Ge}_{15} \mathrm{Te}_{85}$ : density functional, high-energy X-ray and neutron diffraction study. J. Phys-Condens. Mat. 24, 015802 (2012).

36. Clark, S. et al. First principles methods using CASTEP. Z. Kristallogr. 220, 567-570 (2005).

37. Pfrommer, B. G., Cote, M., Louie, S. G. \& Cohen, M. L. Relaxation of crystals with the quasi-Newton method. J. Comput. Phys. 131, 133-140 (1997).

38. Govind, N., Petersen, M., Fitzgerald, G., King-Smith, D. \& Andzelm, J. A generalized synchronous transit method for transition state location. Comput. Mater. Sci. 28, 250-258 (2003).

39. Simpson, R. E. et al. Toward the ultimate limit of phase change in $\mathrm{Ge}_{2} \mathrm{Sb}_{2} \mathrm{Te}_{5}$. Nano. Lett. 10, 414-419 (2010).

\section{Acknowledgements}

The research presented herein was performed under the auspices of the SUTD-MIT International Design Center (IDC) and the 'Designer Chalcogenides' (IDSF1200108OH) research project. R.E.S. also acknowledges support from the SUTD Startup Research Grant SRGEPD2012040. J.K. is grateful for his SUTD-MIT postdoctoral fellowship. J.L. acknowledges support by NSF DMR-1410636 and DMR-1120901. S.W. acknowledges financial support from Ramon y Cajal programme RYC-2013-14838, the Marie Curie Career Integration Grant PCIG12-GA-2013-618487, the Fundacio Privada CELLEX, and the Severo Ochoa Programme SEV-2015-0522. 


\section{Author contributions}

R.E.S. conceived the strained superlattice structure. J.K. performed for the DF/MD calculations. X.Z. grew and characterized the superlattices. E.D. and S.W. performed the fs laser switching measurements. All authors analysed the results and the paper was jointly written.

\section{Additional information}

Supplementary Information accompanies this paper at http://www.nature.com/ naturecommunications

Competing financial interests: The authors declare no competing financial interests.
Reprints and permission information is available online at http://npg.nature.com/ reprintsandpermissions/

How to cite this article: Kalikka, J. et al. Strain-engineered diffusive atomic switching in two-dimensional crystals. Nat. Commun. 7:11983 doi: 10.1038/ncomms11983 (2016).

(c) (i) This work is licensed under a Creative Commons Attribution 4.0 International License. The images or other third party material in this article are included in the article's Creative Commons license, unless indicated otherwise in the credit line; if the material is not included under the Creative Commons license, users will need to obtain permission from the license holder to reproduce the material. To view a copy of this license, visit http://creativecommons.org/licenses/by/4.0/ 